CASES AND OBSERVATIONS,

ILLUSTRATING THE

INFLUENCE OF THE NERVOUS SYSTEM

IN REgULATING

\title{
ANIMAL HEAT.
}

By HENRY EARLE, Eso.

ASSISTANT SURGEON TO ST. BARTHOLOMEW'S HOSPITAL, AND SURGEON TO THE FOUNDLING HOSPITAL.

Read Feb. 20, 1816.

Previously to the interesting experiments published by Mr. Brodie, in the PhilosophicalTransactions for 1811, it was a generally received opinion, that animal temperature depended on the chemical changes which the blood undergoes in the round of circulation. On this supposition a beautiful and apparently satisfactory theory had been constructed. This much esteemed fabric of human reasoning has, however, received a severe shock from the experiments above alluded to, which tend to establish the following facts: that when the brain has been destroyed, animal heat ceases to be generated, notwithstanding the functions of respiration are artificially continued, and ap- 
parently all the chemical changes are produced in the lungs; and further, that an animal thus subjected to artificial respiration, cools more rapidly than one that is simply killed by decapitation, probably in consequence of the circulating blood being exposed to the cold stream of air which is introduced into the lungs. From these facts it appears that nervous influence is essential to the production of animal heat.

Having met with some cases of impaired nervous energy which exhibited phænomena, powerfully illustrative of the above position, I have ventured to submit them to the notice of this Society, conceiving that a strict attention to the alterations in the natural functions, produced by accident or disease, must be considered as one of the least fallacious means by which we may hope to arrive at success in our physiological inquiries. In the present instance, the results arising from comparative experiments on animals, and those drawn from pathological observations, most happily correspond; but although they equally tend to prove the importance af the nervous system in regulating the temperature, we must not lose sight of the action of the circulation on the brain and nerves.

In the second part of this paper I shall offer some further observations on this subject, and illustrate it with instances of increased nervous energy, 
which, together with the first set of cases, will tend to throw some light towards elucidating an obscure but beautiful function in the animal economy; whilst at the same time they will enable us, without much difficulty, to reconcile the discrepancies which appear to exist between the former theory of animal heat, and more recent discoveries.

Should the observations $I$ have made, and the conclusions I have drawn, be deemed erroneous and untenable, I trust the facts at least will be sufficiently important to merit attention.

\section{CASE 1 .}

Thomas Anderson, a mariner, in the month of February, 1812, fell from the main yard into a boat alongside of the ship; he was stunned with the fall, and remained insensible for a considerable time. On recovering, he found that the surgeon had bound up his left arm, in consequence of a fracture of the collar-bone. After six days the bandages were removed, and the limb was found useless and paralysed. For about three weeks after the accident, on any attempt to move the limb, and at times when perfectly quiet, he felt violent pain, which he referred to the extremity of the fingers; but as they and the whole arm were 
perfectly insensible to any impressions, it is probable that the pain was excited where the nerves were injured under the clavicle, and that the percipient mind referred it to the extremities, as is frequently the case after amputation.

This painful stage gradually abated, and the arm remained perfectly palsied and useless. In this state he applied to me, about the end of August. From the history of the case it appeared most probable that the same blow which had fractured the clavicle, had lacerated or crushed the axillary plexus of nerves just as they pass under that bone. The circulation of blood did not appear to have suffered, the pulse at the wrist being synchronous, and equally strong with that of the other limb; yet the temperature was greatly below that of the healthy extremity. As he had never tried electricity, I determined to resort to it, conceiving that it might possibly have a beneficial effect in restoring sensibility. On examining the heat of the limbs previous to the use of electricity, I found it as follows. Left or paralytic hand $70^{\circ}$; bend of the arm $85^{\circ}$; axilla $94^{\circ}$. After drawing strong sparks for about ten minutes, the heat of the hand was increased to $74^{\circ}$; bend of the arm to $88^{\circ}$; axilla to $95^{\circ}$. The temperature of the other hand was $92^{\circ}$.

After using electricity for some days, he said that 
he felt a degree of warmth and tingling which remained for a considerable time after its administration. In ten days I repeated the experiment in a more accurate manner, after placing him on an insulated stool. The temperature this time was:

$$
\begin{aligned}
& \text { Before electricity. After electricity. }
\end{aligned}
$$

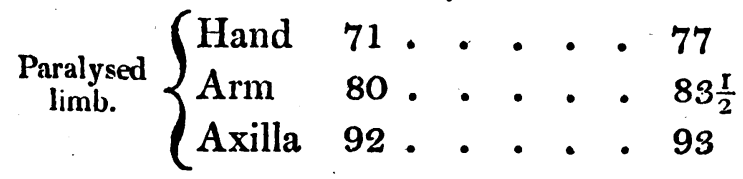

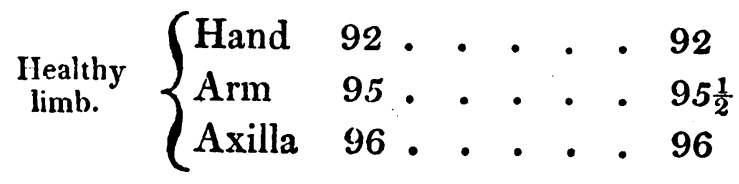

After some time sensation began to return in the integuments about the shoulder and inside of the upper arm; and the muscles of the scapula and great pectoral muscle began to recover their power. It was curious to observe this gradual return of sensibility; one part of the arm possessing natural feeling, another being morbidly sensible, and immediately beyond being quite insensible to every mechanical or chemical injury.

Being desirous of ascertaining if other stimuli acted as powerfully, or if the increased heat depended on any peculiar action of electricity, I determined to apply a blister to the back of the hand. I was obliged to repeat it several times before it would act; at last however a vesication VOL. VII. 
was produced. During the time that the blister was acting, there was no alteration in the thermometer placed immediately contiguous to the edge of the plaster; but on removing the bladder and applying the bulb to the denuded cutis, a rise of three degrees took place. Whether this depended on the stimulus of the blister, or on the removal of the cuticle, by which a more internal part was exposed, I am not able to determine. The blistered surface was not in the least sensible to any injuries, and healed very readily. A short time after this, I recommended him to place his arm in a tub of warm grains, having previously ascertained with his other arm that they were not too hot. I was induced to recommend this remedy, from an idea that the returning sensibility might be aided by raising the temperature to its natural standard, by artificial means. He retained the arm in the pail for nearly half an hour, and on withdrawing it found the whole hand blistered in a most alarming manner, and at the extremities of his fingers and underneath the nails sloughs had formed. He said that he did not at the time feel the grains at all warm, nor did he experience the least pain. A considerable degree of inflammation spread up the absorbents, and matter formed in the axilla, which was soon absorbed and the inflammation assuaged. The temperature of the ulcerated surface of the hand varied from $80^{\circ}$ to $86^{\circ}$, but from the constant application of warm fomentations and poultices, no very accurate result could be obtained, as 
the limb was at all times liable to partake of the degree of heat of surrounding bodies. At the time when the sores were worst, he experienced a heavy aching sensation in the hand, which was not aggravated by any external injuries.

The progress of the -case from this time was slow, but it continued to advance towards a perfect cure. He quitted my care soon after the ulcers were healed, and went on board a ship to act as cook, and I have not since seen or heard from him. When I last examined the limb, the shoulder and upper arm had regained their sensation and power; the integuments in front of the fore-arm were very tender when pressed. The skin on the back of the arm was less sore to the touch. His hand was still insensible, but he felt a consciousness of returning muscular power, and more than once thought that the flexor muscles did contract involuntarily. The temperature of the whole limb was sensibly increased, but the hand was still liable to be affected by the surrounding medium.

On reviewing the circumstances of this case, it appears that a limb deprived of due nervous influence is of a much lower temperature than natural, notwithstanding there is no apparent diminution in the circulation of the blood. That a limb so circumstanced is incapable of supporting any fixed temperature, and is peculiarly liable to partake of the heat of surrounding media; and lastly, that it 
cannot, without injury, sustain a degree of warmth which would not be at all prejudicial to a healthy limb.

\section{CASE II.}

Maria May, aged 14, applied to me in the year 1807 , in consequience of a painful affection of the inside of the fore-arm and hand, extending to the extremity of the little finger. She was unable to account for the origin of the complaint, and said that it had been gradually increasing for some months. At the time of consulting me, the whole course of the ulnar nerve from the elbow downwards, was morbidly sensible when touched; the mere drawing on a glove would sometimes cause so much pain-as to make her scream and fall to the ground. The pain at times occurred spontaneously, without any apparent exciting cause. The integuments on the inside of the fore-arm near the elbow were hotter and fuller than natural. The pain deprived her of rest at night, and her general health was greatly disordered.

She remained under my care for above three years, occasionally suffering extreme torture; at other times being comparatively easy. During this long period a great variety of constitutional and topical remedies were resorted to in vain. During any severe paroxysm she always experienced most 
relief from the application of leeches and cold evaporating poultices. In December, 1810, she was so much worse that I was induced to propose an operation, as the only probable means of affording any permanent benefit. I was well aware how frequently the operation had failed of curing the tic douloureux of the face, but was induced to look for a more favourable termination in the present instance, from the nervous communications being. less numerous, and from the greater length of the nerve, which would enable me to make a division above the diseased part. Having fairly explained the possibility of the disease returning, she readily submitted to the proposed operation, with the prospect of obtaining temporary alleviation from her sufferings. Mr. Langstaff favoured me with his assistance at the operation. I made an incision of about two inches in length, beginning immediately behind the internal condyle of the humerus, and carrying it upwards in the course of the nerve. In making this wound, I exposed a considerable cutaneous branch, which I was afterwards induced to divide, in consequence of the morbid sensibility of the integuments it supplied. Having laid bare about an inch and a half of the nerve, an incision was made through it, at the part nearest the brain; the pain produced was very acute, and felt, to use the girl's own expression, like a violent electrical shock. It was however the last she had to suffer; the little finger and one balf of the ring-finger from that moment lost all sepsation, and the integuments 
on the inside of the hand became perfectly void of feeling. Rather more than an inch of the nerve was dissected out as low down as where it passes behind the internal condyle. The neurilema covering the nerve appeared firmer and thicker than natural.

Her health mended rapidly, after this source of irritation was removed; she lost all her nervous sensations; the wound healed readily, and in about three weeks from the operation she was perfectly well. All the parts supplied by the ulnar nerve had lost their sensibility, and the little finger remained in a paralysed useless state.

A short time after, during severe frosty weather, she called on me in consequence of a blister having formed on the little finger, at the extremity of which, and under the nail, there was a slough. She was unable to account for this, unless from the severity of the weather; that finger being at all times much colder than any other part of the body. By keeping it constantly wrapped up in a warm linseed poultice, it soon healed.

Three several times after this she applied to me with a similar affection, arising apparently from sudden alternations of temperature, as the weather continued cold, and she was obliged to wash dishes in warm water of a temperature not at all unpleasant to the rest of the hand. I regret that I did 
not then accurately ascertain the comparative heat of the finger and the rest of the hand; but at that time my attention was not alive to the subject, and I contented myself with noting down the phænomenon *.

From the circumstances of this case I conceive it adgiissible to conclude, that the want of power of supporting such variations of temperature as were perfectly harmless to the rest of the hand, was dependent on the want of nervous energy; as this was the only apparent deviation from a healthy natural state.

This opinion is greatly strengthened by reading

- Since writing this paper, I have had an opporturfity of seeing my patient, and of making an examination of the state of her hand. The little finger still remains nearly useless; she can bend. it when the other fingers are bent, but possesses little or no poswer over it, independent of the others. She feels when severely pinched or injured, but her sensation in it is still very imperfect; and it conveys a.wrong impression of the form of bodies and of their temperature.

It is always colder than the rest of the hand; on examining it with a thermometer I found that when the bulb was applied to the outside of the root of the little finger it stood at $56^{\circ}$; between the roots of the little and ring finger $57^{\circ}$; outer side of the forefinger $60^{\circ}$; between the fore-finger and_thumb, and in the palm of the hand $62^{\circ}$. The heat of the other hand was on the surface of the different fingers $60^{\circ}$; between the roots of the finger, and in the palm $62^{\circ}$. The temperature of the room was $55^{\circ}$.

It is now January 20th, 1816,-just five years since the operation was performed. 
a highly interesting case of impaired nervous energy, accompanied with a loss of power of regulating the animal temperature, which was published by Dr. Yelloly in the Third Volume of the Transactions of this Society, to which I must refer for any further particulars.

As a further illustration of deficient nervous power, accompanied with a loss of temperature, I may mention, that in examining paralytic limbs I have invariably found them colder than any other part of the body, unless they have been kept artificially warm. Some time since a friend examined twenty-five cases in the Bath Hospital, and found the paralysed limbs in every instance below the natural standard.

In every case in which I have had an opportunity of making any inquiries, where the nervous energy has been materially impaired, the power of maintaining a healthy standard temperature has been in a greater or less degree lost, notwithstanding the circulation of the blood has been apparently unaltered, either in degree or quantity; clearly, in my opinion, proving the important share which the brain and nerves have in regulating and producing animal heat, and shewing, in a remarkable manner, that a perfect integrity of the nervous system is requisite to enable the body to resist the extraordinary variations of temperature to which it has been at times exposed, and to maintain under 
these different circumstances a standard heat of its own, with scarcely any perceptible thermometrical change.

I shall now proceed to consider briefly, the phænomena produced on the nervous system by the changes which the blood undergoes in the round of circulation, and endeavour to ascertain how far such changes may be supposed to contribute to the production of animal heat; by observing the effect of any morbid alterations either in the quantity or quality of the blood, in augmenting or diminishing the temperature *. Previously, however, to entering on this subject, it will be right to premise, that it is not my intention to discuss the chemical nature of the changes effected by respiration, but simply to observe the sensible results of such alterations on the nervous system; as I do not conceive that it is of much importance to the present question, whether or not the venous and arterious blood contain the same proportions of oxygen, but in different states of chemical combination. It is suffi. cient to know that important changes do take place during the transmission of blood through the

* The experiments of Dr. Davy, on the comparative heat of arterial and venous blood, prove that the temperature of the former is greater than that of the latter, and that the temperature of the left side of the heart is greater than that of the right. Here then we have demonstrative evidence of the acquisition of heat during. the pulmonary circulation; we must therefore consider the circulation of the blood as one squrce of animal temperature. 
lungs, and that such changes are essential to the support of nervous action.

It is well known that when from any cause the proper supply of arterial blood is suddenly arrested, and the brain deprived of its wonted stimulus, syncope ensues, accompanied with a great diminution in the warmth of the body. In malformations of the heart; in the blue child for instance, and in all cases of difficult transmission of blood, whether from disease in the lungs or sanguiferous system, the nervous functions are more or less deranged; such persons being very liable to faint, and being at all times subject to great coldness in the extremities and whole body, accompanied with numbness and imperfect sensation. These facts are so well known that it is not necessary to dwell further upon them.

On the other hand, where the circulation of the blood is morbidly increased as in fever, the nervous functions are greatly deranged by the overexcitement, and generally during a paroxysm the temperature of the body is increased, in some degree, in proportion to the violence of the other symptoms. When a sudden or great determination of arterious blood takes place to the nerves of any part, it is known to produce a local affection similar to the more general derangement caused by determination to the brain. This is the case in 
many instances of amaurosis, which are remediable by copious bleedings.

The painful sensation produced by suddenly heating the hands after much exposure to cold, may likewise be referred to the rapid flow of blood over exciting the nerves. When this takes place to a great degree, and when the alternation of temperature has been very sudden, inflammation and sometimes mortification is the consequence; on this principle we apply cold to a frost-bitten limb, and gradually increase the heat to prevent the effect of a sudden over excitement of a part whose vital powers have been exhausted.

In no case, perhaps, is the effect of a local determination more marked than in tic douloureux. In every instance of this distressing malady which has fallen under my observation, during each paroxysm of pain, there was an evident increased flow of blood to the part, accompanied with an increase of heat more or less perceptible.

In the instance of Maria May related above, this was well marked. In a very interesting case which I have lately attended, of affection of the nerves of the forehead and face, there was a well defined red line in the whole course of the supraorbitary nerve, accompanied with so much heat as rapidly to evaporate any cold washes which were applied. 
In another case of a middle-aged widow lady, who had been afflicted for years with tic doulouireux of the inferior maxillary nerve, for the cure of which she had submitted to several operations, but who still at times suffered from pain in the deep seated temporal branches, the gustatory nerve, and all the branches supplying the masseter, pterygoid and buccinator muscles; during each paroxysm there was a violent pulsation in all the branches of the external carotid artery, which terminated generally in a most profuse flow of saliva, after which she experienced temporary relief.

In all these affections, the local abstraction of blood and the application of cold are found most beneficial, and occasionally immediate ease is obtained, by forcibly compressing the part, and thus diminishing the influx of blood. A curious instance in illustration of this was lately mentioned to me. A blacksmith, who for many years had suffered from a violent pain in the nerves of the forehead whenever he exerted himself, accidentally found that he obtained ease by compressing the trunks of the temporal arteries; upon which he contrived a strong spring with a pad at each end, which he fixed on his head in such a manner as to compress the temporal arteries. This spring he wore whenever he went to work, and was thus enabled to remain at the anvil all day without suffering. 
That there is an increased flow of blood to the part in these painful nervous affections, is not only shown by the redness and arterial action, but has been proved by an interesting dissection mentioned by Bichat, of a case of painful affection of the sciatic nerve, where the vessels of the neurilema were evidently enlarged in size, and increased in number, so as to be quite tortuous.

A singular instance, illustrating the effect of an inflammatory determination of blood to a nerve, occurred to me in the course of last year, and tends much to corroborate the preceding remarks.

Maria Williams, a foundling, aged 32, who had been retained in the hospital in consequence of a deficiency of intellect, in February, 1814, wounded her arm with a fork, and punctured the external cutaneous nerve, about half way down the forearm. She experienced much pain soon after the accident, in the whole course of the nerve, and considerable inflammation took place in the neighbourhood of the wound. She was directed to keep the arm very quiet, and to apply evaporating washes. About three weeks after the accident she had occasion to use the limb, when she was suddenly attacked with great pain and a sense of burning in the seat of the original wound. Erysepelatous inflammation soon spread over the whole front of the fore-arm, which terminated in several 
large vesications, giving an appearance very similar. to the disease termed Pemphigus. The heat of the arm was very great, and quickly dried the damp cloths which were applied. By perfect rest, and evaporating poultices with opium, she soon recovered from this attack; but on attempting shortly after to use the arm, a recurrence of the same symptoms took place. The heat was so great this time as to lead me to ascertain its extent with a thermometer, when $I$ found that the mercury rose nearly three degrees higher when applied to the arm, than when placed under the tongue. At this time vesication had taken place in some parts, and probably the temperature was lower than it had been in the previous stage of inflammation. Subsequently to this, she experienced four several relapses, all apparently induced by inadvertently using the arm. The last attack was in September, and differed somewhat in its character from the former; no vesications following the inflammation, and the appearance bearing more analogy to urticaria than to pemphigus.

The inflammation was always confined to the front of the fore-arm, and did not appear disposed to spread. The nerve during the whole time was acutely sensible when pressed. After September she retained her arm constantly in a sling for the space of three months, and has experienced no return of inflammation since. 
The increased temperature in this case was very remarkable, and forms a striking contrast with the first case which I have related of crushed nerve, where the thermometer only rose to $74^{\circ}$ when applied to the exposed cutis of a recently blistered surface. I consider this case as equally important in illustrating the action of arterious blood on the nerves, and in explaining the phænomena of inflammation. It is also strongly corroborative of the observations of Sir E. Home, detailed in some experiments, which he published in the Second Part of the Philosophical Transactions for the year 1814, proving the influence of the nerves upon the action of the arteries; for I conceive there can be no doubt that these violent inflammatory symptoms were all produced by the injury done to the nerve, as they could at any time be brought on by exciting it in any way.

It appears then, from the evidence which has been adduced, in the first place, that an integrity of the nervous system is essential to the evolution of animal heat, and that when any part of it is impaired by accident or disease, the due performance of this calorific function is either partially or generally deranged. Secondly, that the stimulus of arterious blood is necessary for the excitement of the brain and nerves, and likewise for the proper developement' of animal heat. Thirdly, that there exists a considerable sympathy between the 
nervous and sanguiferous systems, and that an injury inflicted on a nerve is attended with an increased arterial action, and a local determination of blood. Lastly, that such a local determination, whether the effect of disease or injury is accompanied by a sensible increase of temperature at the part.

In conclusion, I shall offer some remarks on the curious phænomenon produced by placing a ligature on the principal artery of a limb, which has hitherto been difficult to account for, but which admits of a ready solution on the principle of vital heat, being the result of the action of arterious blood on the nerves, (a conclusion which the facts related above would seem to warrant) whilst at the same time it affords a powerful illustration in support of such a theory.

When a ligature is placed on the principal artery supplying a limb with blood, the circulation in the smaller anastomosing vessels, and in the capillary system is much increased. The limb is furnished with a smaller quantity of blood, but what does circulate must necessarily pass through vessels of a smaller calibre, consequently they are preternaturally distended with blood; and if a limb be examined under these circumstances, it will be found that the communicating vessels are enlarged. They subsequently undergo a further change, and after 
some time again contract to their former size. The effect produced by these changes on the tempera. ture of the limb; is an increase of heat beyond the natural standard of the healthy limb, at that part immediately below where the artery is tied, which increase of heat gradually extends itself over the whole limb. This could not happen if the temperature depended solely on the circulation, as the actual volume of blood, the supposed source of heat, is lessened ; but at the same time be it remembered, that the smaller order of vessels and the capillaries, in short those vessels which immediately supply the nerves, are receiving more than a due proportion, consequently the nervous system is overexcited and more heat developed, first, at that part where there is the greatest impulse of blood, and subsequently over the whole limb, in proportion as the increased collateral circulation is established.

It may be urged in opposition to this explanation, that this increased temperature does not invariably follow the application of a ligature to the trunk of an artery. It will not be difficult to reply to such objections, when it is considered under what very different circumstances a ligature is applied, and that in many instances of aneurism the collateral circulation is in a great degree developed before the ligature is applied ; in others, the main trunk below the aneurismal sac is obliterated; and in others again, a principal communicating vessel of the first order is given off a short distance above 
the situation of the ligature; all which circumstances would very materially influence the results, for reasons too obvious to require explanation. In all the instances, however, in which I have known a ligature suddenly applied to the main trunk of an artery, in a limb which had not undergone any previous change, the increased temperature above described has been the invariable consequence.

Berners Street, Dec. 28, 1815. 